\title{
AMENABLE PURELY INFINITE ACTIONS ON THE NON-COMPACT CANTOR SET
}

\author{
GÁBOR ELEK
}

\begin{abstract}
We prove that any countable non-amenable group $\Gamma$ admits a free minimal amenable purely infinite action on the non-compact Cantor set. This answers a question of Kellerhals, Monod and Rørdam [10].
\end{abstract}

Keywords. Minimal actions, non-compact Cantor set, topological amenability, purely infinite actions

\section{Contents}

1. Introduction 2

2. Non-compact Bernoulli subshifts 3

3. Landscapes and minimality 4

4. Hilly landscapes and Borel hyperfiniteness 6

5. Landscapes with rivers 7

6. The Cantor code for amenability 9

7. The combinatorial version of paradoxicality 11

8. Paradoxicalization 12

9. The proof of Theorem $1 \quad 14$

10. A remark about actions on the compact Cantor set 16

$\begin{array}{ll}\text { References } & 16\end{array}$

2010 Mathematics Subject Classification. 46L55.

The author was partially supported by the ERC Consolidator Grant "Asymptotic invariants of discrete groups, sparse graphs and locally symmetric spaces" No. 648017. 


\section{INTRODUCTION}

Free, minimal (topological) actions on the non-compact Cantor set $\mathbf{K}^{*}$ were constructed by Matui and Rørdam [11] and Danilenko [4] (see also [3]). Furthermore, the action constructed in [4] is amenable (even Borel-hyperfinite). In [10] Kellerhals, Monod and Rørdam studied free minimal amenable actions on $\mathbf{K}^{*}$ that are purely infinite as well. They showed that for such actions the associated reduced $C^{*}$-algebra is always a stable Kirchberg algebra of the UCT class. Let us recall the notion of a purely infinite action on $\mathbf{K}^{*}$ from [10] [Definition 4.4]. Let $\alpha: \Gamma \curvearrowright \mathbf{K}^{*}$ be an action of a countable group $\Gamma$ on the non-compact Cantor set. Recall that $\mathbf{K}^{*}$ is the unique (up to homeomorphisms) locally compact, non-compact, totally disconnected, metrizable Hausdorff space that contains no isolated points. We say that a compact-open set $K$ is paradoxical with respect to the action if there exist pairwise disjoint compact-open sets $K_{1}, K_{2}, \ldots, K_{n+m}$ and elements $t_{1}, t_{2}, \ldots, t_{n+m} \in \Gamma$ such that $K_{j} \subset K$ for all $j$ and

$$
K=\cup_{i=1}^{n} \alpha\left(t_{i}\right)\left(K_{i}\right)=\cup_{j=n+1}^{n+m} \alpha\left(t_{j}\right)\left(K_{j}\right) .
$$

The action $\alpha$ is called purely infinite if all the compact-open subsets of $\mathbf{K}^{*}$ are paradoxical with respect to the action. In [10] Kellerhals, Monod and Rørdam proved that if a countable group $\Gamma$ contains an exact nonsupramenable subgroup, then $\Gamma$ admits a free minimal amenable purely infinite action on $\mathbf{K}^{*}$. They asked whether any non-supramenable group admits such an action. The goal of this paper is to give a positive answer for this question by proving the following theorem.

Theorem 1. Every countable non-amenable group $\Gamma$ admits a free minimal amenable purely infinite action on $\mathbf{K}^{*}$.

Note that Theorem 1 combined with the above mentioned result in [10] implies that a countable group admits a free minimal amenable purely infinite action on $\mathbf{K}^{*}$ if and only if it is non-supramenable. We will prove the theorem for finitely generated groups and use the fact (Lemma 7.1 in [10]) that if a group $\Gamma$ contains a subgroup $H$ which admits such free minimal amenable purely infinite action, then the group $\Gamma$ itself admits an action with the same properties as well. By the same reason, we do not need to consider the case of non-supramenable amenable groups. The strategy of the proof goes as follows. In Section 2 we introduce the notion of non-compact Bernoulli shifts. Some special elements of these spaces are called proper landscapes. In Section 3 we show that the orbit closure of a proper landscape always contains an invariant subset $Y$ that is homeomorphic to $\mathbf{K}^{*}$ and the Bernoulli action of the group on $Y$ is both free and minimal. It is not hard to construct landscapes such that the resulting free minimal action is Borel hyperfinite, but these actions cannot be extended to purely infinite actions. So, we will construct free minimal actions $\beta$ on $\mathbf{K}^{*}$ such that $\mathbf{K}^{*}$ can be exhausted by compacta that admit free group actions from the topological full group of $\beta$. Using the fact that the free group has Yu's Property A we construct an amenable 
extension of $\beta$. Then, with the help of the partial actions of the free group and the well-known paradoxical property of non-amenable graphs we inductively construct a sequence of extensions for which more and more compact-open sets are becoming paradoxical, in such a way that freeness, minimality and amenability are preserved. In the resulting free minimal amenable limit action all the compact-open sets will be paradoxical and this will finish the proof of our theorem.

\section{NON-COMPACT BerNOUlli SUBSHIFTS}

For the rest of the paper let $\Gamma$ be a finitely generated group with a symmetric generating set $\Sigma=\left\{\sigma_{i}\right\}_{i=1}^{n}$. Let $A=\{0,1\}^{\mathbb{N}} \times\{\mathbb{N} \cup\{\infty\}\}$. We equip $\{0,1\}^{\mathbb{N}}$ with the standard product topology and we regard the space $\{\mathbb{N} \cup\{\infty\}$ as the compactification of the natural numbers. Hence, $A$ is a totally disconnected space homeomorphic to the Cantor set. We consider the Bernoulli space $A^{\Gamma}$. Clearly, $A^{\Gamma}$ is homeomorphic to the Cantor set as well and $\Gamma$ acts (on the left) on $A^{\Gamma}$ continuously by translations, that is, $L_{\gamma}(x)(\delta)=x(\delta \gamma)$. If $x \in A^{\Gamma}$ and $x(\gamma)=(a, b)$, then we refer to $a$ as the Cantor coordinate $C(x(\gamma))$ of $x(\gamma)$ and to $b$ as the height coordinate $H(x(\gamma))$ of $x(\gamma)$. A minimal non-compact Bernoulli subshift is

- a $\Gamma$-invariant subset $Y \subset A^{\Gamma}$ such that $Y$ is homeomorphic to $K^{*}$;

- and for every element $y \in Y$ the orbit of $y$ is dense in $Y$.

An element $y \in A^{\Gamma}$ is of totally finite height if for all $\gamma \in \Gamma$ the height coordinate of $y(\gamma)$ is a finite number. We say that $y \in A^{\Gamma}$ is of totally infinite height if for all $\gamma \in \Gamma$ the height coordinate of $y(\gamma)$ is $\infty$. We call $y \in A^{\Gamma}$ of totally finite height a regular element if the orbit closure of $y$ consists only of elements of totally finite height and of totally infinite height. Our first goal is to give a sufficient condition for a regular element $y \in A^{\Gamma}$ having such orbit closure $\bar{O}(y)$ that the totally finite height part of $\bar{O}(y)$ is a free, minimal, non-compact Bernoulli subshift.

Let us consider the (right) Cayley graph $G=\operatorname{Cay}(\Gamma, \Sigma)$ of our group $\Gamma$ equipped with the shortest distance metric $d_{G}$. A labeling $\lambda: \Gamma \rightarrow\{0,1\}^{\mathbb{N}}$ is called a proper Cantor labeling if the following condition holds. For every $r>0$ there exists $S_{r}>0$ such that if $0<d_{G}(\gamma, \delta) \leq r$ then $(\lambda(\gamma))_{S_{r}} \neq$ $(\lambda(\delta))_{S_{r}}$, where $(x)_{s} \in\{0,1\}^{s}$ denotes the first $s$ coordinates of the element $x \in\{0,1\}^{\mathbb{N}}$.

Proposition 2.1. There exist proper labelings on $\Gamma$.

Proof. Let $d$ be the degree of the vertices of $G$. Then, for all $k \geq 1$ we have a function

$$
\lambda_{k}: \Gamma \rightarrow\left\{1,2, \ldots, d^{k}+1\right\}
$$

such that if $0<d_{G}(\gamma, \delta) \leq k$ then

$$
\lambda_{k}(\gamma) \neq \lambda_{k}(\delta)
$$


Let $\zeta_{k}: \Gamma \rightarrow\{0,1\}^{d^{k}+1}$ be defined by

$$
\zeta_{k}(\gamma)=(0,0, \ldots, 1, \ldots, 0,0)
$$

where the only 1 is at the $\lambda_{k}(\gamma)$-th position. Now we can define the proper labeling by

$$
\lambda(\gamma)=\left(\zeta_{1}(\gamma) \zeta_{2}(\gamma) \ldots\right)
$$

Lemma 2.1. Let $y \in A^{\Gamma}$ be an element such that the Cantor coordinates of $y$ amount to a proper Cantor labeling of $\Gamma$. Then the action of $\Gamma$ on the orbit closure of $y$ is free.

Proof. Let $x \in \bar{O}(y)$. Then the Cantor coordinates of $x$ also amount to a proper Cantor labeling of $\Gamma$. Consequently, the Cantor coordinates of $x$ are all different. Hence if $e_{\Gamma} \neq \gamma \in \Gamma$, then $L_{\gamma}(x) \neq x$ and the freeness of the action follows.

Now we introduce our key notion: the landscape. Landscapes are characterized by the height coordinates.

Definition 2.1. Let $y \in A^{\Gamma}$ be an element of totally finite height. We say that $y$ is a landscape if the following four conditions are satisfied.

- If $d_{G}(\gamma, \delta)=1$ then $|H(y(\gamma))-H(y(\delta))| \leq 1$.

- For all $n \geq 1$ there exists $M(y, n)>1$ such that if $H(y(\gamma))=n$ then there exists $\delta, d_{G}(\delta, \gamma) \leq M(y, n)$ so that $H(y(\gamma))=1$.

- for all $l \geq 1$ there exist $N(y, l)>1$ such that if $H(y(\gamma))=1$ then the ball $B_{N(y, l)}(G, \gamma)$ of radius $N(y, l)$ centered at $\gamma$ contains at least $l$ elements $\delta$ for which $H(y(\delta))=1$.

- for all $m \geq 1$, there exists $S(y, m)>1$ so that every ball $B_{S(y, m)}(G, \delta)$ in our graph $G$ contains an element $\kappa$ such that $H(y(\kappa)) \geq m$.

We call a landscape proper if its Cantor coordinates amount to a proper Cantor labeling of $\Gamma$. The following lemma is straightforward.

Lemma 2.2. Landscapes are regular and if $x$ is an element of totally finite height in the orbit closure of a (proper) landscape, then $x$ is a (proper) landscape with the same structure contants as $y$.

\section{LANDSCAPES AND MINIMALITY}

The goal of this section is to prove the following proposition.

Proposition 3.1. Let $y \in A^{\Gamma}$ be a proper landscape. Then the orbit closure of $y$ contains an invariant set $Y_{y} \subset A^{\Gamma}$ homeomorphic to $\mathbf{K}^{*}$ such that the restricted action of $\Gamma$ on $Y_{y}$ is free and minimal.

Proof. For each pair of integers $m, n \geq 1$ we consider the finite set $C U_{\Gamma}^{m, n}$. An element $B$ of $C U_{\Gamma}^{m, n}$ is a labeling of the vertices of the ball $B_{m}\left(G, e_{\Gamma}\right)$ by 
elements of the set

$$
\{0,1\}^{m} \times\{l \in \mathbb{N}|| n-l \mid \leq m\},
$$

in such a way that the second coordinate of the label of $e_{\Gamma}$ equals to $n$. Let $x \in A^{\Gamma}$ be a proper landscape. For each $m \geq 1$, we have a map

$$
\Theta_{x}^{m}: \Gamma \rightarrow \cup_{n=1}^{\infty} C U_{\Gamma}^{m, n}
$$

constructed in the following way. First of all, $\Theta_{x}^{m}(\gamma)$ will be an element of $C U_{\Gamma}^{m, H(x(\gamma))}$. Let $\delta \in B_{m}\left(G, e_{\Gamma}\right)$. Then, $\Theta_{x}^{m}(\gamma)(\delta)=(a, b)$, where

- $a=(C(x(\gamma \delta)))_{m}$.

- $b=H(x(\gamma \delta))$.

Let $\mathcal{B}$ denote the countable set $\cup_{m=1}^{\infty} \cup_{n=1}^{\infty} C U_{\Gamma}^{m, n}$. For each $x \in A^{\Gamma}$, we have a partition $\mathcal{B}=I_{x} \cup J_{x} \cup K_{x}$, where

- $K_{x}$ is the subset of labeled balls $B$ in $\mathcal{B}$ such that $\Theta_{x}^{m}(\gamma) \neq B$ if $\gamma \in \Gamma$. Here $m$ is the radius of $B$.

- $J_{x}$ is the subset of labeled balls $B$ in $\mathcal{B}$ such that there exists $K_{B}>0$ so that if $H(x(\gamma))=1$, then there exists $\delta, d_{G}(\delta, \gamma) \leq K_{B}$ for which $\Theta_{x}^{m}(\delta)=B$. Again, $m$ is the radius of $B$.

- $I_{x}$ is defined as $\mathcal{B} \backslash\left(K_{x} \cup J_{x}\right)$.

The following lemma is trivial.

Lemma 3.1. If $x$ is a proper landscape and $x^{\prime}$ is an element of totally finite height in the orbit closure of $x$, then

- $K_{x^{\prime}} \supseteq K_{x}$.

- $J_{x^{\prime}} \supseteq J_{x}$.

- $I_{x^{\prime}} \subseteq I_{x}$.

Lemma 3.2. Let $x \in A^{\Gamma}$ be a a proper landscape and $B \in I_{x}$ be a labeled ball of radius $m$. Then there exists a proper landscape $x^{\prime}$ in the orbit closure of $x$ such that $B \in K_{x^{\prime}}$ and $H\left(x^{\prime}\left(e_{\Gamma}\right)\right)=1$.

Proof. By the definition of $I_{x}$, we have a sequence $\left\{\gamma_{k}\right\}_{k=1}^{\infty}$ such that $H\left(x\left(\gamma_{k}\right)\right)=1$ and if $d_{G}\left(\delta, \gamma_{k}\right) \leq k$ then $\Theta_{x}^{m}(\delta) \neq B$. Let $x^{\prime} \in A^{\Gamma}$ be the limit point of the sequence $\left\{L_{\gamma_{k}}(x)\right\}_{k=1}^{\infty}$ in $A^{\Gamma}$. Note that such limit point must exist by the landscape conditions and $x^{\prime}$ is again a proper landscape. Then $B \in K_{x^{\prime}}$ and $H\left(x^{\prime}\left(e_{\Gamma}\right)\right)=1$.

Lemma 3.3. Let $y \in A^{\Gamma}$ be a proper landscape. Then we have an element $z \in A^{\Gamma}$ in the orbit closure of $y$ such that

- $H\left(z\left(e_{\Gamma}\right)\right)=1$.

- The set $I_{z}$ is empty.

(we will call such elements $z \in A^{\Gamma}$ minimal) 
Proof. Let $I_{y}=\left\{B_{1}, B_{2}, \ldots \ldots\right\}$. Using Lemma 3.1 and 3.2, we can inductively construct a sequence $\left\{y_{n}\right\}_{n=1}^{\infty}$ in the orbit closure of $y$ such that

- $H\left(y_{n}\left(e_{\Gamma}\right)\right)=1$ and

- $B_{i} \notin I_{y_{n}}$ if $1 \leq i \leq n$.

Let $z$ be a limit point of the sequence $\left\{y_{n}\right\}_{n=1}^{\infty}$. Then $z$ is a proper landscape and the set $I_{z}$ is empty.

Now we can finish the proof of our proposition. Let $z \in A^{\Gamma}$ be the minimal proper landscape in the previous lemma. The invariant subspace $Y_{y}$ is defined as the set of elements of totally finite height in the orbit closure $\bar{O}(z)$. By Lemma 2.2, all other elements of $\bar{O}(z)$ are of totally infinite height. Let $t \in Y_{y}$. It is enough to prove that $\bar{O}(t)$ contains $z$, that is, for all $m \geq 1$ there exists $y_{m} \in \bar{O}(t)$ such that $\Theta_{z}^{m}\left(e_{\Gamma}\right)=\Theta_{y_{m}}^{m}\left(e_{\Gamma}\right)$. Let $\Theta_{z}^{m}\left(e_{\Gamma}\right)=B_{m}$. Since $B_{m} \in J_{z}$ there exists $K_{m}>0$ such that if $H(z(\delta))=1$, then there exists $\gamma$ so that

- $d_{G}(\delta, \gamma) \leq K_{m}$ and

- $\Theta_{z}^{m}(\gamma)=B_{m}$.

Since $t \in \bar{O}(z)$, if $H(t(\delta))=1$ then there exists some $\rho_{m} \in \Gamma$ such that $d_{G}\left(\rho_{m}, \delta\right) \leq K_{B}$ and $\Theta_{t}^{m}\left(\rho_{m}\right)=B_{m}$. That is, $\Theta_{y_{m}}^{m}\left(e_{\Gamma}\right)=B_{m}$ if $y_{m}=L_{\rho_{m}}(t)$.

Finally, we need to show that $t$ is not an isolated point in $Y_{y}$. Let $\Theta_{t}^{m}\left(e_{\Gamma}\right)=$ $B_{m}^{\prime}$. By our third landscape condition and the minimality of $z$, there exists $e_{\Gamma} \neq \gamma_{m} \in \Gamma$ such that $\Theta_{t}^{m}\left(\gamma_{m}\right)=B_{m}^{\prime}$ as well. Hence, $\Theta_{L_{\gamma_{m}}(t)}^{m}=B_{m}^{\prime}$. Since by freeness $L_{\gamma_{m}}(t) \neq t$ for all $m \geq 1$, we have that $t$ is not an isolated point.

\section{Hilly landscapes AND Borel hyperfiniteness}

Let $z \in A^{\Gamma}$ be a proper landscape. We say that $z$ is hilly if for all $n \geq 1$ there exists $Q_{n}$ such that the induced graph in $G$ on the set $W_{z}^{n} \subset \Gamma$, where

$$
W_{z}^{n}=\{\gamma \mid H(z(\gamma)) \leq n\}
$$

has components of size at most $Q_{n}$. Clearly, if $y$ is a minimal landscape in the orbit closure of a hilly landscape $z$ then $y$ is hilly as well (with the same structure constants $\left.\left\{Q_{n}\right\}_{n=1}^{\infty}\right)$. Let $\alpha: \Gamma \curvearrowright X$ be a Borel action of $\Gamma$ on a Borel space $X$. We say that $p, q \in X$ are equivalent, $p \equiv_{E} q$, if for some $\gamma \in \Gamma, \alpha(\gamma)(p)=q$. The equivalence relation $E$ is called the orbit equivalence relation of the action. Recall that $\alpha$ is called Borel hyperfinite, if $E$ is the increasing union of some finite Borel equivalence relations $E_{1} \subset E_{2} \subset \ldots$.

Proposition 4.1. Let $y$ be a minimal hilly landscape. Then the action of $\Gamma$ on the totally finite part $Y$ of the orbit closure of $y$ is Borel hyperfinite (consequently amenable, see Section 6).

Proof. We define the finite equivalence relation $E_{n}$ on $Y$ in the following way. If $t, s \in Y$ then $t \equiv_{E_{n}} s$ if 
- either $t=s$,

- or $L_{\gamma}(t)=s$ for some $e_{\Gamma} \neq \gamma \in \Gamma$, such that there exists a path $\left(e_{\Gamma}=\gamma_{1}, \gamma_{2}, \ldots, \gamma_{l}=\gamma\right)$ in $G$ for which $t\left(\gamma_{i}\right) \leq n$ holds if $1 \leq i \leq l$.

Since the elements of $Y$ are hilly, $E_{n}$ is indeed a finite Borel equivalence relation. Clearly, $E_{1} \subset E_{2} \subset \ldots$ and $\cup_{n=1}^{\infty} E_{n}$ is the orbit equivalence relation on $Y$. Therefore the action of $\Gamma$ on $Y$ is Borel hyperfinite.

Proposition 4.2. There are hilly landscapes on $\Gamma$.

Proof. We use a fractal-like construction to build the landscape (one should note that the so-called $(C, F)$ - construction in [3] also has a fractal-like character). So, let $A_{0}=\left\{e_{\Gamma}\right\}, A_{1}=A_{0} \cup\left\{\gamma_{1}\right\}$, where $d_{G}\left(e_{\Gamma}, \gamma_{1}\right)=30$. Let $A_{2}=A_{1} \cup$ $\gamma_{2} A_{1}$, where $d_{G}\left(e_{\Gamma}, \gamma_{2}\right)=300$ and inductively, let $A_{n}=A_{n-1} \cup \gamma_{n} A_{n-1}$, where $d_{G}\left(e_{\Gamma}, \gamma_{n}\right)=3\left(10^{n}\right)$. Let $A=\cup_{n=1}^{\infty} A_{n}$. Observe that any non-unit element of $A$ can be uniquely written as $\gamma_{n_{k}} \gamma_{n_{k-1}} \ldots \gamma_{n_{1}}$, where $n_{k}>n_{k-1}>\cdots>n_{1}$. We define the subsets

$$
A \supset Q_{1} \supset Q_{2} \supset \ldots
$$

by

$$
Q_{n}:=\left\{e_{\Gamma}\right\} \cup\left\{\delta \mid \delta=\gamma_{n_{k}} \gamma_{n_{k-1}} \ldots \gamma_{n_{1}} \text { and } n_{1} \geq n\right\} .
$$

So, in particular $\gamma_{n} \in Q_{n}$. Observe that

- If $\gamma, \delta \in Q_{n}$ then $B_{10^{n}}(G, \delta)$ and $B_{10^{n}}(G, \gamma)$ are disjoint.

- If $\gamma \in Q_{k}$ and $\delta \in Q_{l}$, where $k<l$ then either $B_{10^{k}}(G, \gamma) \subset B_{10^{l}}(G, \delta)$ or $B_{10^{k}}(G, \gamma) \cap B_{10^{l}}(G, \delta)=\emptyset$.

Now we can define the landscape $z$ on $\Gamma$ in the following way. Let $H(z(\gamma))=l$ if

- $\gamma \in B_{10^{l}}(\delta)$ for some $\delta \in Q_{l}$ and also

- $\gamma \notin B_{10^{k}}(\rho)$ if $\rho \in Q_{k}$ and $k<l$.

Also, let $\gamma \rightarrow C(z(\gamma))$ be an arbitrary proper labeling. It is easy to check that $z$ is, in fact, a hilly landscape.

Remark 4.1. We can construct an explicit hilly landscape on the group of integers $Z$ using the construction above. Call a non-negative integer $n$ ternary if all the digits of $n$ are 0 or $3: 0,3,30,33,300,303, \ldots$ We only need to define $H: \mathbb{Z} \rightarrow \mathbb{N}$. Let $H(n)=1$ if $n$ is a ternary number. In general, let $H(n)=k$, if $k$ is the smallest non-negative integer such that $|n-t| \leq 10^{k}$, where $t$ is a ternary number and $10^{k}$ divides $t$.

\section{LANDSCAPES WITH RIVERS}

The Borel hyperfinite construction of the previous section cannot be extended to a purely infinite action, so we need a different idea. Let $G$ be the Cayley graph of $\Gamma$ as in the previous sections. Also, let $T$ be the infinite tree for which all the vertex degrees are four (the 4-tree). A river is a bilipschitz 
embedding of the 4-tree $T$ into $G$, that is, a map $\Psi: V(T) \rightarrow \Gamma$ such that there exists some $C>0$ so that for all $x, y \in V(T)$

$$
C^{-1} d_{T}(x, y) \leq d_{G}(\Psi(x), \Psi(y)) \leq C d_{T}(x, y) .
$$

Let us also assume that $H(y(\gamma)):=d_{G}(\Psi(V(T), \gamma))+1$ defines a landscape on $\Gamma$ and $e_{\Gamma} \in \Psi(V(T))$. We call such $y$ a landscape with river.

Proposition 5.1. Landscapes with rivers do exist on non-amenable groups.

Proof. By Theorem 1.5 of [2], bilipschitz embeddings $\Psi_{1}: V(T) \rightarrow \Gamma$ exist. Clearly, the resulting element $y$ would satisfy the first three landscape conditions. However, the fourth condition might not be satisfied, say, because $\Gamma$ is the free group and $\Psi_{1}$ is surjective. So, let us consider a bilipschitz map $\Phi: V(T) \rightarrow V(T)$ such that for all $t \in V(T)$, there is at least one branch $B_{T}$ of $t$ in the tree $T$ so that $B_{T} \cap \Phi(V(T))$ is empty. Let $\Psi=\Psi_{1} \circ \Phi$. We can also assume that $e_{\Gamma} \in \Psi(V(T))$. Then the resulting element $y$ will satisfy the fourth landscape condition as well.

The following proposition will be crucial in the next section. Note that for a set $A, \operatorname{Fin}(A)$ denotes the the family of all finite subsets of $A$.

Proposition 5.2. Let $\Psi: V(T) \rightarrow \Gamma$ be a bilipschitz embedding of the 4 - tree into our Cayley graph $G$ in such a way that $e_{\Gamma} \in \Psi(V(T))$ and

$H(y(\gamma)):=d_{G}(\Psi(V(T)), \gamma)+1$ defines a landscape on $\Gamma$. Let $C>0$ be an integer such that if $x, y \in V(T)$ then

$$
C^{-1} d_{T}(x, y) \leq d_{G}(\Psi(x), \Psi(y)) \leq C d_{T}(x, y) .
$$

Then for all $m \geq 1$ we have a map $\kappa_{m}: \Gamma \rightarrow$ Fin $(\Psi(V(T)))$ such that

- For all $\gamma \in \Gamma,\left|\kappa_{m}(\gamma)\right|=m$.

- For all $\gamma \in \Gamma, \kappa_{m}(\gamma) \subset B_{d_{G}(\Psi(V(T)), \gamma)+C m}(G, \gamma)$.

- If $d_{G}\left(\gamma_{1}, \gamma_{2}\right)=1$, then

$$
\left|\kappa_{m}\left(\gamma_{1}\right) \triangle \kappa_{m}\left(\gamma_{2}\right)\right| \leq 2\left(d_{G}\left(\Psi(V(T)), \gamma_{1}\right)+1\right) C .
$$

Proof. We use the classical construction that shows the 4-tree has Property A. This process hopefully explains why we call these objects rivers. First, let us fix an infinite ray $\left\{t_{i}\right\}_{i=0}^{\infty}$ in $V(T)$. That is, $d_{T}\left(t_{0}, t_{i}\right)=i$ and $d_{T}\left(t_{i-1}, t_{i}\right)=1$. If $s \in V(T)$, then we have a unique path $\left(s_{1}, s_{2}, \ldots, s_{l}\right)$ such that $s=s_{1}, s_{l}=t_{i}$ for some non-negative integer $i$, and $s_{l-1} \notin\left\{t_{i}\right\}_{i=0}^{\infty}$. Then, we consider the infinite path $P(s)=\left(s_{1}, s_{2}, \ldots, s_{l}, s_{l+1} \ldots\right)$, where for all $j \geq 1, s_{l+j}=t_{i+j}$. So, for each $m \geq 1$, we have the path $P_{m}(s)=\left(s_{1}, s_{2}, \ldots, s_{m}\right)$. Then for all $s \in V(T),\left|P_{m}(s)\right|=m$. Also, if $p, q \in V(T)$ and $d_{T}(p, q)=a$, then $\left|P_{m}(p) \triangle P_{m}(q)\right| \leq a$. Now for all $\gamma \in \Gamma$, pick an element $\delta_{\gamma}$ in $\Psi(V(T))$ such that $d_{G}\left(\delta_{\gamma}, \gamma\right)=d_{G}(\Psi(V(T)), \gamma)$ and let

$$
\kappa_{m}(\gamma)=\Psi\left(P_{m}\left(\Psi^{-1}\left(\delta_{\gamma}\right)\right)\right)
$$


Now, if $d_{G}\left(\gamma_{1}, \gamma_{2}\right)=1$, then $d_{G}\left(\delta_{\gamma_{1}}, \delta_{\gamma_{2}}\right) \leq 2\left(d_{G}\left(\Psi(V(T)), \gamma_{1}\right)+1\right)$. Hence, $\left|\kappa_{m}\left(\gamma_{1}\right) \triangle \kappa_{m}\left(\gamma_{2}\right)\right| \leq 2\left(d_{G}\left(\Psi(V(T)), \gamma_{1}\right)+1\right) C$. Also, for all $\gamma \in \Gamma, \kappa_{m}(\gamma) \subset$ $B_{d_{G}(\Psi(V(T)), \gamma)+C m}(G, \gamma)$.

Let $y \in A^{\Gamma}$ be an element of totally finite height such that $H(y(\gamma))=$ $d_{G}(\Psi(V(T)), \gamma)+1$ for some river with bilipschitz constant $C$. Let

$$
H_{1}(y)=\Psi(V(T))=\{\gamma \in \Gamma \mid H(y(\gamma))=1\} .
$$

Let $G_{y}$ be a graph on the vertex set $H_{1}(y)$ defined in the following way.

- $V\left(G_{y}\right)=H_{1}(y)$.

- $(p, q) \in E\left(G_{y}\right)$ if $d_{G}(p, q) \leq C$.

Then $G_{y}$ has bounded vertex degrees and it is quasi-isometric to the 4-tree $T$. In particular, $G_{y}$ has positive Cheeger constant. Recall that the Cheeger constant of an infinite graph $J$ is defined in the following way.

$$
c(J)=\inf _{H \in \operatorname{Fin}(V(J))} \frac{|\partial(H)|}{|H|},
$$

where $\partial(H)=\left\{p \in H \mid \exists q \notin H, d_{J}(p, q)=1\right\}$. Now let $z \in A^{\Gamma}$ be an element of totally finite height in the orbit closure of $y$. We can construct the graph $G_{z}$ on $H_{1}(z)=\{\gamma \in \Gamma \mid H(z(\gamma))=1\}$ as above.

Lemma 5.1. The graph $G_{z}$ is connected and is of bounded vertex degree. Also, $G_{z}$ has positive Cheeger constant, in fact, $c\left(G_{z}\right) \geq c\left(G_{y}\right)$.

Proof. Since $K_{z} \subseteq K_{z^{\prime}}$ it is clear that $G_{z}$ has bounded vertex degrees and $c\left(G_{z}\right) \geq c\left(G_{y}\right)$. Now we prove the connectivity of $G_{z}$. Let $\gamma, \gamma \delta \in H_{1}(z)$ such that $\delta \in B_{m}\left(G, e_{\Gamma}\right)$. Note that if $p, q \in H_{1}(y)$ and $d_{G}(p, q) \leq m$, then $d_{G_{y}}(p, q) \leq C m$. Since $z$ is in the orbit closure of $y$, there exist $\rho, \rho \delta \in H_{1}(y)$ such that

$$
\Theta_{z}^{C^{2} m}(\gamma)=\Theta_{y}^{C^{2} m}(\rho)
$$

Since $\rho$ and $\rho \delta$ can be connected by a path in $H_{1}(y)$ inside the ball $B_{C^{2} m}(G, \rho)$ we can conclude that $\gamma$ and $\gamma \delta$ can be connected by a path in $H_{1}(z)$ inside the ball $B_{C^{2} m}(G, \gamma)$. This finishes the proof our lemma.

\section{The Cantor code for amenability}

Let $y$ be a proper landscape with river (so we also assume that a proper labeling $\gamma \rightarrow C(y(\gamma))$ is given) and for each $m \geq 1$ let $\kappa_{m}: \Gamma \rightarrow \operatorname{Fin}(\Psi(V(T)))$ be the map as in Section 5. That is, for all $\gamma \in \Gamma$

$$
\kappa_{m}(\gamma) \subset B_{F_{m, n}}(G, \gamma)
$$

where $n=H(y(\gamma))=d_{G}(\Psi(V(T)), \gamma)$ and $F_{m, n}=C m+n$. For $\gamma \in \Gamma$, let $L_{m}(\gamma) \subset B_{F_{m, n}}\left(G, e_{\gamma}\right)$ be the subset such that

$$
\gamma L_{m}(\gamma)=\kappa_{m}(\gamma)
$$


For each $m, n \geq 1$ let $\left\{a_{1}^{m, n}, a_{2}^{m, n}, \ldots, a_{\tau_{m, n}}^{m, n}\right\}$ be an enumeration of the finite subsets of $B_{F_{m, n}}\left(G, e_{\Gamma}\right)$, where $\tau_{m, n}=2^{\left|B_{F_{m, n}}\left(G, e_{\Gamma}\right)\right|}$. So, for each $\gamma \in \Gamma$ we have an element $c_{\gamma} \in\{0,1\}^{\mathbb{N}}$ constructed in the following way. Let $L_{m}(\gamma)=a_{i_{m, n, \gamma}}^{m, n}$, where $1 \leq i_{m, n, \gamma} \leq \tau_{m, n}$. Now let $c_{m, \gamma}$ be the concatenation of $i_{m, n, \gamma}$ pieces of the string 010. Let

$$
c_{\gamma}=\left(11 c_{1, \gamma} 11 c_{2, \gamma} 11 c_{3, \gamma} 11 \ldots\right) \in\{0,1\}^{N} .
$$

Therefore, for each $\gamma \in \Gamma$ we have two elements of the Cantor set:

$$
C(y(\gamma))=\left(u_{1}^{\gamma} u_{2}^{\gamma} \ldots\right)
$$

and

Let $z \in A^{\Gamma}$ be defined by

$$
c_{\gamma}=\left(v_{1}^{\gamma} v_{2}^{\gamma} \ldots\right)
$$

- $H(z(\gamma))=H(y(\gamma))$.

- $C(z(\gamma))=\left(u_{1}^{\gamma} v_{1}^{\gamma} u_{2}^{\gamma} v_{2}^{\gamma} u_{3}^{\gamma} v_{3}^{\gamma} \ldots\right)$

Clearly, $z$ is a proper landscape. Notice that $z$ encodes the landscape $y$ and for each $m \geq 1$, the system $\left\{\kappa_{m}(\gamma)\right\}_{\gamma \in \Gamma}$. Finally, let $x$ be a minimal element in the orbit closure of $z$ and $Y$ be the totally finite part of the orbit closure of $x$.

Proposition 6.1. The action of $\Gamma$ on $Y$ is free, minimal and amenable.

Proof. By Proposition 3.1, freeness and minimality follow. So, let us recall the definition of amenable actions on locally compact spaces.

Definition 6.1. [1] Let $\Gamma$ be a finitely generated group with a finite generating system $\Sigma$. Let $\alpha: \Gamma \curvearrowright X$ be a continuous action of $\Gamma$ on the locally compact space $X$. The action $\alpha$ is topologically amenable if there exists a sequence $\left\{g_{m}: X \times \Gamma \rightarrow \mathbb{R}\right\}_{m=1}^{\infty}$ of non-negative functions such that

- For all $m \geq 1$ and $p \in X, \sum_{\gamma \in \Gamma} g_{m}(p, \gamma)=1$.

- for all generator $\sigma \in \Sigma$

$$
\sum_{\gamma \in \Gamma}\left|g_{m}(\alpha(\sigma)(p), \sigma \gamma)-g_{m}(p, \gamma)\right|
$$

uniformly tends to zero on the compact subsets of $X$.

Let $t \in Y$. Then

$$
C(t(\gamma))=\left(u_{t, \gamma}^{1} v_{t, \gamma}^{1} u_{t, \gamma}^{2} v_{t, \gamma}^{2} \ldots\right)
$$

Let us consider

$$
C_{v}(t(\gamma))=\left(v_{t, \gamma}^{1}, v_{t, \gamma}^{2} \ldots\right)
$$

By our construction,

$$
C_{v}(t(\gamma))=\left(11 d_{t, \gamma}^{1} 11 d_{t, \gamma}^{2} 11 \ldots\right),
$$

where $d_{t, \gamma}^{m}$ is the concatenation of $j_{m, t, \gamma}$ pieces of the string 010. Also, $j_{m, t, \gamma} \leq$ $\tau_{m, H(t(\gamma))}$. Let us define $g_{m}: Y \times \Gamma \rightarrow \mathbb{R}$ in the following way. Let $g_{m}(t, \rho)=\frac{1}{m}$ 
if $\rho \in a_{j_{m, t, e_{\Gamma}}^{m}, H\left(t\left(e_{\Gamma}\right)\right)}$, otherwise, let $g_{m}(t, \rho)=0$. Clearly, $g_{m}$ is continuous and for all $t \in Y, \sum_{\rho \in \Gamma} g_{m}(t, \rho)=1$. Since $Y$ is contained in the orbit closure of the element $z$, for all $t \in Y$ there exists $\delta \in \Gamma$ such that

- $H(z(\delta))=H\left(t\left(e_{\Gamma}\right)\right)$.

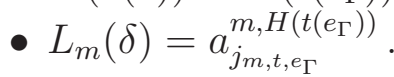

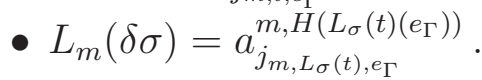

Therefore by (1),

$$
\sum_{\rho \in \Gamma}\left|g_{m}\left(L_{\sigma}(t), \sigma \rho\right)-g_{m}(t, \rho)\right| \leq \frac{2\left(H\left(t\left(e_{\Gamma}\right)\right)+2\right) C}{m} .
$$

That is,

$$
\sum_{\rho \in \Gamma}\left|g_{m}\left(L_{\sigma}(t), \sigma \rho\right)-g_{m}(t, \rho)\right|
$$

uniformly tends to zero on the set $Y_{n}=\left\{t \in Y \mid H\left(t\left(e_{\gamma}\right)\right) \leq n\right\}$. Since for all compact open set $K \subset Y$ there exists $n \geq 1$ such that $K \subset Y_{n}$, our proposition follows.

\section{THE COMBINATORIAL VERSION OF PARADOXICALITY}

Let $z \in A^{\Gamma}$ be a minimal proper landscape and for $m \geq 1$ let $\Theta_{z}^{m}: \Gamma \rightarrow$ $\cup_{n=1}^{\infty} C U_{\Gamma}^{m, n}$ be the map defined in Section 3. We say that the subset $T \subseteq \Gamma$ is z-local if there exists $m \geq 1$ and a finite subset $S \subset \cup_{n=1}^{\infty} C U_{\Gamma}^{m, n}$ such that $T=\left(\Theta_{z}^{m}\right)^{-1}(S)$. Notice that the $z$-locality of the subset $T$ means that the membership of $T$ can be locally verified. We call a $z$-local subset $T z$ paradoxical if there exist pairwise disjoint $z$-local subsets $T_{1}, T_{2}, \ldots, T_{p+q}$ and elements $\gamma_{1}, \gamma_{2}, \ldots, \gamma_{p+q} \in \Gamma$ such that $T_{j} \subset T$ for all $j$, and

$$
T=\cup_{i=1}^{p} T_{i} \gamma_{i}=\cup_{j=p+1}^{p+q} T_{j} \gamma_{j} .
$$

Now let $Z$ be the totally finite height part of the orbit closure $\bar{O}(z)$ of $z$. We define the map $\Theta_{Z}^{m}: Z \rightarrow \cup_{n=1}^{\infty} C U_{\Gamma}^{m, n}$ by

$$
\Theta_{Z}^{m}(x)=\Theta_{x}^{m}\left(e_{\Gamma}\right) .
$$

Note that $\Theta_{Z}^{m}$ is a locally constant function, hence if $S \subset \cup_{n=1}^{\infty} C U_{\Gamma}^{m, n}$ is a finite subset then $\left(\Theta_{Z}^{m}\right)^{-1}(S)$ is a compact-open subset of the locally compact space $Z$. Moreover, by the definition of the product topology, any compactopen subset $U$ of $Z$ can be written as $\left(\Theta_{Z}^{m}\right)^{-1}(S)$ for some $m \geq 1$ and finite subset $S \subset \cup_{n=1}^{\infty} C U_{\Gamma}^{m, n}$. The key observations of this section are the following propositions.

Proposition 7.1. Let $m \geq 1$ and let $S \subset \cup_{n=1}^{\infty} C U_{\Gamma}^{m, n}$ be a finite subset. Let $z \in A^{\Gamma}$ be a minimal proper landscape and $Z$ be as above. Suppose that the $z$-local subset $T=\left(\Theta_{z}^{m}\right)^{-1}(S)$ is z-paradoxical. Then $U=\left(\Theta_{Z}^{m}\right)^{-1}(S)$ is a paradoxical compact-open subset of $Z$. Consequently, if all z-local subsets of $\Gamma$ are $z$-paradoxical then the action of $\Gamma$ on $Z$ is purely infinite. 
Proof. Let $\gamma_{1}, \gamma_{2}, \ldots, \gamma_{n+m} \in \Gamma$ such that $T_{j} \subset T$ for all $j$ and

$$
T=\cup_{i=1}^{p} T_{i} \gamma_{i}=\cup_{j=p+1}^{p+q} T_{j} \gamma_{j} .
$$

Then there exists $l>m$ and $S_{1}, S_{2}, \ldots, S_{n+m} \in \operatorname{Fin}\left(\cup_{n=1}^{\infty} C U_{\Gamma}^{l, n}\right)$ such that for all $1 \leq i \leq p+q, T_{i}=\left(\Theta_{z}^{l}\right)^{-1}\left(S_{i}\right)$. Now observe that

$$
U=\cup_{i=1}^{p} L_{\gamma_{i}}\left(U_{i}\right)=\cup_{j=p+1}^{p+q} L_{\gamma_{j}}\left(U_{j}\right),
$$

where $U_{i}=\left(\Theta_{Z}^{l}\right)^{-1}\left(S_{i}\right)$. Hence, $U$ is indeed paradoxical.

Proposition 7.2. Let $z \in A^{\Gamma}$ be a landscape and let $w \in A^{\Gamma}$ be an element of totally finite height in the orbit closure of $z$. Let $m>0$ and let $S \in$ $\operatorname{Fin}\left(\cup_{n=1}^{\infty} C U_{\Gamma}^{m, n}\right)$. Also, let $l>m$, for $1 \leq i \leq p+q$ let $S_{i} \in \operatorname{Fin}\left(\cup_{n=1}^{\infty} C U_{\Gamma}^{l, n}\right)$ and $\gamma_{1}, \gamma_{2}, \ldots, \gamma_{p+q} \in \Gamma$ such that

- $T=\left(\Theta_{z}^{m}\right)^{-1}(S), T_{i}=\left(\Theta_{z}^{l}\right)^{-1}\left(S_{i}\right)$.

- $T_{i} \subset T$ for $1 \leq i \leq p+q$.

- $T=\cup_{i=1}^{p} T_{i} \gamma_{i}=\cup_{j=p+1}^{p+q} T_{j} \gamma_{j}$.

Then the sets $\left\{T_{i}^{w}\right\}_{i}^{p+q}$ are disjoint, $T_{i}^{w} \subset T^{w}$ and $T^{w}=\cup_{i=1}^{p} T_{i}^{w} \gamma_{i}=$ $\cup_{j=p+1}^{p+q} T_{j}^{w} \gamma_{j}$, where $T^{w}=\left(\Theta_{w}^{m}\right)^{-1}(S), T_{i}=\left(\Theta_{w}^{l}\right)^{-1}\left(S_{i}\right)$. That is, $T^{w}$ is $w$ paradoxical (note that empty sets are paradoxical by definition).

Proof. Let $a$ be an integer such that $\gamma_{1}, \gamma_{2}, \ldots, \gamma_{n+m} \in B_{a}\left(G, e_{\Gamma}\right)$. First, let us prove that $T_{i}^{w} \cap T_{j}^{w}=\emptyset$ if $i \neq j$. Suppose that $\gamma \in T_{i}^{w} \cap T_{j}^{w}$. Since $w$ is in the orbit closure of $z$, there exists $\delta \in \Gamma$ such that $\Theta_{z}^{l}(\delta)=\Theta_{w}^{l}(\gamma)$. Hence, $\delta \in T_{i} \cap T_{j}$ leading to a contradiction. Now let $\gamma \in T_{i}^{w}$. We need to show that $\gamma \gamma_{i} \in T^{w}$. Again, we have $\delta \in \Gamma$ such that $\Theta_{z}^{a+l+m}(\delta)=\Theta_{w}^{l+a+m}(\gamma)$. Then $\delta \in T_{i}$, so $\delta \gamma_{i} \in T$, hence $\gamma \gamma_{i} \in T^{w}$. Finally, let $\gamma \in T^{w}$. Let us show that there exists $1 \leq i \leq p$ such that $\gamma \gamma_{i}^{-1} \in T_{i}^{w}$ and $p+1 \leq j \leq p+q$ such that $\gamma \gamma_{j}^{-1} \in T_{j}^{w}$. Again, let $\delta \in \Gamma$ such that $\Theta_{z}^{a+l+m}(\delta)=\Theta_{w}^{l+a+m}(\gamma)$. Then $\delta \in T$, hence for some $1 \leq i \leq p$ and $p+1 \leq j \leq p+q$ we have that $\delta \gamma_{i}^{-1} \in T_{i}$ and $\delta \gamma_{j}^{-1} \in T_{j}$. Thus, $\gamma \gamma_{i}^{-1} \in T_{i}^{w}$ and $\gamma \gamma_{j}^{-1} \in T_{j}^{w}$.

\section{Paradoxicalization}

Let $z, z^{\prime} \in A^{\Gamma}$ be landscapes and let $l \geq 1$ be an integer. Then $z \equiv_{l} z^{\prime}$ if for all $\gamma \in \Gamma$ :

- $C(z(\gamma))_{l}=C\left(z^{\prime}(\gamma)\right)_{l}$.

- If $C(z(\gamma))=\left(a_{1} b_{1} a_{2} b_{2} \ldots\right)$ and $C\left(z^{\prime}(\gamma)\right)=\left(c_{1} d_{1} c_{2} d_{2} \ldots\right)$, then for all $n \geq 1, a_{n}=c_{n}$.

- $H(z(\gamma))=H\left(z^{\prime}(\gamma)\right)$.

The following lemma is a straightforward consequence of the definition.

Lemma 8.1. Let $z \in A^{\Gamma}$ be a landscape, let $m>0$ and $S \in F i n\left(\cup_{n=1}^{\infty} C U_{\Gamma}^{m, n}\right)$. Also, let $l>m$ and for $1 \leq i \leq p+q$, let $S_{i} \in \operatorname{Fin}\left(\cup_{n=1}^{\infty} C U_{\Gamma}^{l, n}\right)$ and 
$\gamma_{1}, \gamma_{2} \ldots, \gamma_{p+q} \in \Gamma$ such that $\emptyset \neq T$ is z-paradoxical and

$$
T=\left(\Theta_{z}^{m}\right)^{-1}(S)=\cup_{i=1}^{p} T_{i} \gamma_{i}=\cup_{j=p+1}^{p+q} T_{j} \gamma_{j},
$$

where $T_{i}=\left(\Theta_{z}^{l}\right)^{-1}\left(S_{i}\right)$. Then if $z \equiv_{r} z^{\prime}$, where $r \geq l: T$ is $z^{\prime}$-paradoxical as well.

One of main ingredients of the proof of Theorem 1 is the following proposition.

Proposition 8.1. Let $z$ be a minimal landscape and let $\emptyset \neq T=\left(\Theta_{z}^{m}\right)^{-1}(S)$ be a z-local set, where $S \in F i n\left(\cup_{n=1}^{\infty} C U_{\Gamma}^{m, n}\right)$. Let $m \leq m^{\prime}$. Then there exists $z^{\prime} \in A^{\Gamma}$ such that $z^{\prime} \equiv_{m^{\prime}} z$ and $T$ is $z^{\prime}$-paradoxical.

Proof. Since $z$ is minimal there exists some $R_{T}>1$ such that if $\gamma \in \Gamma$ then $B_{R_{T}}(G, \gamma) \cap T \neq \emptyset$. Let us construct a graph $G_{T}$ with vertex set $T$ in the following way. The vertices $p, q \in T$ are adjacent in $G_{T}$ if and only if $d_{G}(p, q) \leq$ $3 R_{T}$. It is easy to see that $G_{T}$ is a connected graph with bounded vertex degrees and $G_{T}$ is quasi-isometric to $G$. Since $G$ is the Cayley graph of a non-amenable group, $G$ has positive Cheeger constant. So, since $G$ and $G_{T}$ are quasi-isometric, $G_{T}$ has positive Cheeger constant as well (Theorem 18.13 [6]). Therefore, by the main result of [5] $G_{T}$ is a paradoxical graph. That is, there exist injective maps $\varphi: T \rightarrow T, \psi: T \rightarrow T$ and $K>0$ such that

- $\varphi(T) \cap \psi(T)=\emptyset$.

- For every $x \in T, d_{G}(x, \varphi(x))<K, d_{G}(x, \psi(x))<K$.

Therefore, there exist elements $\gamma_{1}, \gamma_{2}, \ldots, \gamma_{p+q} \in \Gamma$ such that for any $x \in T$

- there exists $1 \leq i \leq p$ such that $\varphi(x) \gamma_{i}=x$,

- there exists $p+1 \leq j \leq q$ such that $\psi(x) \gamma_{j}=x$.

For $1 \leq i \leq p$ let

$$
T_{i}=\left\{y \in T \mid \text { there exists } x \in T \text { such that } \varphi(x) \gamma_{i}=x\right\} .
$$

For $p+1 \leq j \leq p+q$ let

$$
T_{j}=\left\{y \in T \mid \text { there exists } x \in T \text { such that } \psi(x) \gamma_{j}=x\right\} .
$$

Then $T_{1}, T_{2}, \ldots T_{p+q}$ are disjoint sets and

$$
T=\cup_{i=1}^{p} T_{i} \gamma_{i}=\cup_{p+1}^{p+q} T_{j} \gamma_{j} .
$$

We need to construct $z^{\prime} \in A^{\Gamma}$ such that $z \equiv_{m^{\prime}} z^{\prime}$ for all $1 \leq i \leq p+q, T_{i}$ is $z^{\prime}$-local. Let $a_{1}<a_{2}<\cdots<a_{n+m}$ be consecutive even numbers such that $m^{\prime}<a_{1}$. For $\gamma \in \Gamma$ let $H\left(z^{\prime}(\gamma)\right)=H(z(\gamma))$ and

- If $\gamma \notin \cup_{i=1}^{p+q} T_{i}$, then for all $1 \leq i \leq p+q$ let the $a_{i}$-th Cantor coordinate of $z^{\prime}(\gamma)$ be 0 .

- If $\gamma \in T_{j}$ then let the $a_{j}$-th Cantor coordinate of $z^{\prime}(\gamma)$ be 1 and if $i \neq j$ then let the $a_{i}$-th Cantor coordinate of $z^{\prime}(\gamma)$ be 0 . 
For $l>a_{p+q}$ the $l$-th Cantor coordinates of the elements of $\Gamma$ will be chosen in such a way to make $z^{\prime}$ proper. For $l<a_{1}$ let the $l$-th Cantor coordinate of $z^{\prime}(\gamma)$ be equal to the $l$-th Cantor coordinate of $z(\gamma)$. It is easy to see that for the resulting proper landscape $z^{\prime}, z \equiv_{m^{\prime}} z$ and for all $1 \leq i \leq p+q, T_{i}$ is $z^{\prime}$-local.

\section{The proof of Theorem 1}

Now we are in the position to prove our theorem. First, let $\left\{S_{i}\right\}_{i=1}^{\infty}$ be an enumeration of the set $\cup_{m=1}^{\infty} \operatorname{Fin}\left(\cup_{n=1}^{\infty} C U_{\Gamma}^{m, n}\right)$ and $S_{i} \in \operatorname{Fin}\left(\cup_{n=1}^{\infty} C U_{\Gamma}^{m, n}\right)$. Let $y$ be a minimal landscape with a river. Let $z$ and $x$ be the landscapes as in Proposition 6.1. Finally, we define $t_{0} \in A^{\Gamma}$ in the following way.

- For all $\gamma \in \Gamma, H\left(t_{0}(\gamma)\right)=H(x(\gamma))$.

- For $\gamma \in \Gamma$, if $C(x(\gamma))=\left(u_{1} u_{2} u_{3} \ldots\right)$, let $C(t(\gamma))=\left(u_{1} 0 u_{2} 0 u_{3} 0 \ldots\right)$.

We define $\pi_{\text {odd }}: A^{\Gamma} \rightarrow A^{\Gamma}$ as follows. For $p \in A^{\Gamma}$ and $\gamma \in \Gamma$

- $H\left(\pi_{\text {odd }}(p)(\gamma)\right)=H(p(\gamma))$.

- If $C(p(\gamma))=\left(v_{1} v_{2} v_{3} v_{4} v_{5} \ldots\right)$ then $C\left(\pi_{\text {odd }}(p)(\gamma)\right)=\left(v_{1} v_{3} v_{5} \ldots\right)$.

So, $\pi_{\text {odd }}\left(t_{0}\right)=x$ and $t_{0}$ is again a minimal landscape. Now we start our inductional process.

Step 0. If $\left(\Theta_{t_{0}}^{m_{1}}\right)^{-1}\left(S_{1}\right)$ is empty then let $l_{1}=m_{1}, p_{1}=0, q_{1}=1, S_{1}^{1}=\emptyset$, $\gamma_{1}=e_{\Gamma}, t_{1}=t_{0}$. If $\left(\Theta_{t_{0}}^{m_{1}}\right)^{-1}\left(S_{1}\right)$ is a (non-empty) $t_{0}$-local set, then let $t_{0}^{\prime} \in A^{\Gamma}$ be such that $t_{0}^{\prime} \equiv_{m_{1}} t_{0}$ and $S_{1}$ is $t_{0}^{\prime}$-paradoxical (Proposition 8.1). Also, let $\gamma_{1}^{1}, \gamma_{2}^{1}, \ldots, \gamma_{p_{1}+q_{1}}^{1} \in \Gamma$ and $S_{1}^{1}, S_{2}^{1}, \ldots, S_{p_{1}+q_{1}}^{1} \in \operatorname{Fin}\left(\cup_{n=1}^{\infty} C U^{l_{1}, n}\right)$ such that

- For all $1 \leq i \leq p_{1}+q_{1},\left(\Theta_{t_{0}^{\prime}}^{l_{1}}\right)^{-1}\left(S_{i}^{1}\right) \subset\left(\Theta_{t_{0}^{\prime}}^{m_{1}}\right)^{-1}\left(S_{1}\right)$.

- The sets $\left\{\left(\Theta_{t_{0}^{\prime}}^{l_{1}}\right)^{-1}\left(S_{i}^{1}\right)\right\}_{i=1}^{p_{1}+q_{1}}$ are disjoint.

- $\left(\Theta_{t_{0}^{\prime}}^{m_{1}}\right)^{-1}\left(S_{1}\right)=\cup_{i=1}^{p_{1}}\left(\left(\Theta_{t_{0}^{\prime}}^{l_{1}}\right)^{-1}\left(S_{i}^{1}\right)\right) \gamma_{i}^{1}=\cup_{j=p_{1}+1}^{p_{1}+q_{1}}\left(\left(\Theta_{t_{0}^{\prime}}^{l_{1}}\right)^{-1}\left(S_{j}^{1}\right)\right) \gamma_{j}^{1}$.

Finally, let $t_{1}$ be a minimal landscape in the orbit closure of $t_{0}^{\prime}$. Then,

- For all $1 \leq i \leq p_{1}+q_{1},\left(\Theta_{t_{1}}^{l_{1}}\right)^{-1}\left(S_{i}^{1}\right) \subset\left(\Theta_{t_{1}}^{m_{1}}\right)^{-1}\left(S_{1}^{1}\right)$.

- The sets $\left\{\left(\Theta_{t_{1}}^{l_{1}}\right)^{-1}\left(S_{i}^{1}\right)\right\}_{i=1}^{p_{1}+q_{1}}$ are disjoint.

- $\left(\Theta_{t_{1}}^{m_{1}}\right)^{-1}\left(S_{1}^{1}\right)=\cup_{i=1}^{p_{1}}\left(\left(\Theta_{t_{1}}^{l_{1}}\right)^{-1}\left(S_{i}^{1}\right)\right) \gamma_{i}^{1}=\cup_{j=p_{1}+1}^{p_{1}+q_{1}}\left(\left(\Theta_{t_{1}}^{l_{1}}\right)^{-1}\left(S_{j}^{1}\right)\right) \gamma_{j}^{1}$.

So,

- $t_{1}$ is a minimal landscape.

- $\pi_{\text {odd }}\left(t_{1}\right)$ is in the orbit closure of $x$.

- $\left(\Theta_{t_{1}}^{m_{1}}\right)^{-1}\left(S_{1}\right)$ is $t_{1}$-paradoxical.

Step k. Suppose that we have a minimal landscape $t_{k}$ and we also have

- For all $1 \leq a \leq k, \gamma_{1}^{a}, \gamma_{2}^{a}, \ldots, \gamma_{p_{a}+q_{a}}^{a} \in \Gamma$. 
- For all $1 \leq a \leq k, S_{1}^{a}, S_{2}^{a}, \ldots, S_{p_{a}+q_{a}}^{a} \in \operatorname{Fin}\left(\cup_{n=1}^{\infty} C U^{l_{a}, n}\right)$ for some $m_{a}<l_{a}$.

such that

- $\pi_{\text {odd }}\left(t_{k}\right)$ is in the orbit closure of $x$.

- For all $1 \leq a \leq k$ and $1 \leq i \leq p_{a}+q_{a},\left(\Theta_{t_{k}}^{l_{a}}\right)^{-1}\left(S_{i}^{a}\right) \subset\left(\Theta_{t_{k}}^{m_{a}}\right)^{-1}\left(S_{a}\right)$.

- For all $1 \leq a \leq k$, the sets $\left\{\left(\Theta_{t_{k}}^{l_{a}}\right)^{-1}\left(S_{i}^{a}\right)\right\}_{i=1}^{p_{a}+q_{a}}$ are disjoint.

- For all $1 \leq a \leq k,\left(\Theta_{t_{k}}^{m_{a}}\right)^{-1}\left(S_{a}\right)=\cup_{i=1}^{p_{a}}\left(\left(\Theta_{t_{k}}^{l_{a}}\right)^{-1}\left(S_{i}^{a}\right)\right) \gamma_{i}^{a}=$ $\cup_{j=p_{a}+1}^{p_{a}+q_{a}}\left(\left(\Theta_{t_{k}}^{l_{a}}\right)^{-1}\left(S_{j}^{a}\right)\right) \gamma_{j}^{a}$.

Now, in the same way as in Step 0 . we construct a minimal landscape $t_{k+1}$ and elements $\gamma_{1}^{k+1}, \gamma_{2}^{k+1}, \ldots, \gamma_{p_{k+1}+q_{k+1}}^{k+1} \in \Gamma$ and sets $S_{1}^{k+1}, S_{2}^{k+1}, \ldots, S_{p_{k+1}+q_{k+1}}^{k+1} \in$ $\operatorname{Fin}\left(\cup_{n=1}^{\infty} C U^{l_{k+1}, n}\right)$ for some $m_{k+1}<l_{k+1}$ in such a way that

- $\pi_{\text {odd }}\left(t_{k+1}\right)$ is in the orbit closure of $x$.

- For all $1 \leq a \leq k+1$ and $1 \leq i \leq p_{a}+q_{a},\left(\Theta_{t_{k+1}}^{l_{a}}\right)^{-1}\left(S_{i}^{a}\right) \subset$ $\left(\Theta_{t_{k+1}}^{m_{a}}\right)^{-1}\left(S_{a}\right)$.

- For all $1 \leq a \leq k+1$, the sets $\left\{\left(\Theta_{t_{k+1}}^{l_{a}}\right)^{-1}\left(S_{i}^{a}\right)\right\}_{i=1}^{p_{a}+q_{a}}$ are disjoint.

- For all $1 \leq a \leq k+1,\left(\Theta_{t_{k+1}}^{m_{a}}\right)^{-1}\left(S_{a}\right)=\cup_{i=1}^{p_{a}}\left(\left(\Theta_{t_{k+1}}^{l_{a}}\right)^{-1}\left(S_{i}^{a}\right)\right) \gamma_{i}^{a}=$ $\cup_{j=p_{a}+1}^{p_{a}+q_{a}}\left(\left(\Theta_{t_{k+1}}^{l_{a}}\right)^{-1}\left(S_{j}^{a}\right)\right) \gamma_{j}^{a}$.

Then we have a subsequence $k_{1}<k_{2}<\ldots$ such that $\lim _{r \rightarrow \infty} t_{k_{r}}=t \in A^{\Gamma}$ exists.

Proposition 9.1. All t-local subset of $\Gamma$ is t-paradoxical.

Proof. Let $b \geq 1$ such that $\left(\Theta_{t}^{m_{b}}\right)^{-1}\left(S_{b}\right)$ is non-empty. We need to show that

(1) For all $1 \leq i \leq p_{b}+q_{b},\left(\Theta_{t}^{l_{b}}\right)^{-1}\left(S_{i}^{b}\right) \subset\left(\Theta_{t}^{m_{b}}\right)^{-1}\left(S_{b}\right)$.

(2) The sets $\left\{\left(\Theta_{t}^{l_{b}}\right)^{-1}\left(S_{i}^{b}\right)\right\}_{i=1}^{p_{b}+q_{b}}$ are disjoint.

(3) $\left(\Theta_{t}^{m_{b}}\right)^{-1}\left(S_{b}\right)=\cup_{i=1}^{p_{b}}\left(\left(\Theta_{t}^{l_{b}}\right)^{-1}\left(S_{i}^{b}\right)\right) \gamma_{i}^{b}=\cup_{j=p_{b}+1}^{p_{b}+q_{b}}\left(\left(\Theta_{t}^{l_{b}}\right)^{-1}\left(S_{j}^{b}\right)\right) \gamma_{j}^{b}$.

Now we proceed in the same way as in the proof of Proposition 7.2. Let us prove (1). Let $c>0$ be an integer such that $\gamma_{1}^{b}, \gamma_{2}^{b} \ldots \gamma_{p_{b}+q_{b}}^{b} \in B_{c}\left(G, e_{\gamma}\right)$ Let $\gamma \in\left(\Theta_{t}^{l_{b}}\right)^{-1}\left(S_{i}^{b}\right)$. We need to show that $\gamma \gamma_{i}^{b} \in\left(\Theta_{t}^{m_{b}}\right)^{-1}\left(S_{b}\right)$. Since $t=$ $\lim _{r \rightarrow \infty} t_{k_{r}}$ we have $k_{r}>b$ such that $\Theta_{t_{k_{r}}}^{c+l_{b}+m_{b}}(\gamma)=\Theta_{t}^{c+l_{b}+m_{b}}(\gamma)$. Then $\gamma \in$ $\left(\Theta_{t_{k_{r}}}^{l_{b}}\right)^{-1}\left(S_{i}^{b}\right)$, so $\gamma \gamma_{i}^{b} \in\left(\Theta_{t_{k_{r}}}^{m_{b}}\right)^{-1}\left(S_{b}\right)$, hence $\gamma \gamma_{i}^{b} \in\left(\Theta_{t}^{m_{b}}\right)^{-1}\left(S_{b}\right)$. The proof of (2) and (3) can be obtained in a similar fashion.

Observe that $\pi_{\text {odd }}(t)$ is in the orbit closure of $x$. Let $\hat{t}$ be a minimal landscape in the orbit closure of $t$ and let $Y$ be the totally finite part of the orbit closure of $\hat{t}$. Then $\pi_{\text {odd }}(\hat{t})$ is in the orbit closure of $x$ as well. Hence, by Proposition 6.1 the action of $\Gamma$ on $Y$ is free, minimal and amenable. Also, by Proposition 7.2 all $\hat{t}$-local subsets of $\Gamma$ are $\hat{t}$-paradoxical. Hence, by Proposition 7.1 the action of $\Gamma$ on $Y$ is purely infinite. Since by Proposition $3.1 Y$ is homeomorphic to $\mathbf{K}^{*}$, our theorem follows. 


\section{A REMARK about aCtions on the COMPACT CANTOR SET}

If $\Gamma$ is a non-amenable group then one can consider the compact Bernoulli subshift $X=C^{\Gamma}$, where $C=\{0,1\}^{\mathbb{N}}$. Repeating the arguments of our paper one can construct a free, minimal purely infinite $\Gamma$-subshift $Y$ in $X$ such that $Y$ is homeomorphic to the Cantor set. If the group is exact, then using the witness-sets for Property A as in Section 5 one can even make the action amenable. This result is originally due to Rørdam and Sierakowski [12]. Our method just helps to avoid the use of the Cech-Stone compactification. Similarly, one can eliminate the Cech-Stone compactification from the proof of Theorem 1.3. (iv) in [9] and add pure infinity to the properties of the action. That is, one can obtain (using the witness-sets of finite asymptotic dimension) the following result: All countable non-amenable group $\Gamma$ of asymptotic dimension $d$ has a free, minimal, purely infinite action of dynamic asymptotic dimension at most $d$. One can also extend all these results for uniformly recurrent subgroups [8] as well. Let $\Gamma$ be a finitely generated group and be $H \subset \Gamma$ a subgroup such that the orbit closure of $H$ in $\operatorname{Sub}(\Gamma)$ is a closed, invariant, minimal subspace. That is, $Z=\bar{O}(H)$ is a uniformly recurrent subgroup (URS). If the Schreier graph $\Gamma / H$ is non-amenable (that is the URS $\mathrm{Z}$ is not coamenable [7]) then using the method of our paper we can construct a free minimal purely infinite $Z$-proper (nonfree) action of $\Gamma$ (see $[7]$ for the definition of $Z$-properness). If the Schreier graph is of Property $A$ then we can even assume that the action is topologically amenable.

\section{REFERENCES}

[1] C. Anantharaman-Delaroche, Amenability and exactness for dynamical systems and their $C^{*}$-algebras. Trans. Amer. Math. Soc. 354 (2002), 4153-4178.

[2] I. Benjamini And O. Schramm, Every graph with a positive Cheeger constant contains a tree with a positive Cheeger constant. Geom. Funct. Anal. 7 (1997), no. 3, 403-419.

[3] A.I. Danilenko, On simplicity concepts for ergodic actions. J. Anal. Math. 102, (2007), $77-117$.

[4] A.I. Danilenko, Rank-one actions, their $(C, F)$-models and constructions with bounded parameters. preprint: arXiv:1610.09851

[5] W.A. Deuber, M. Simonovits and V.T. Sós, A note on paradoxical metric spaces. Studia Sci. Math. Hungar. 30, (1995), 17-23.

[6] C. Drutu And M. Kapovich, Geometric group theory. With an appendix by Bogdan Nica. Amer. Math. Soc. Coll. Publ. 63 Amer. Math.Soc. (2018)

[7] G. EleK, Uniformly recurrent subgroups and simple $C^{*}$-algebras. Journal of Funct. Anal. 274 (2018) no.6 1657-1689.

[8] E. Glasner and B. Weiss, Uniformly recurrent subgroups. Recent trends in ergodic theory and dynamical systems. Contemp. Math., 631, Amer. Math. Soc., Providence, RI, 2015. 63-75.

[9] E. Guentner, R. Willett and G. Yu, Dynamical asymptotic dimension: relation to dynamics, topology, coarse geometry and $C^{*}$-algebras. Math. Ann. 367 (2017) no.1$2,785-829$.

[10] J. Kellerhals, N. Monod ANd M. RøRdAm, Non-supramenable groups acting on locally compact spaces. Doc. Math. 18, (2013), 1597-1626. 
[11] H. Matui AND M. RøRDAm, Universal properties of group actions on locally compact spaces. J. Funct. Anal. 268, (2015), no. 12, 3601-3648.

[12] M. RøRdam And A. Sierakowski, Purely infinite $C^{*}$-algebras arising from crossed products. Ergodic Theory Dynam. Systems 32 (2012), no. 1, 273-293.

Department of Mathematics And Statistics, Fylde College, Lancaster UniVERsity, Lancaster, LA1 4YF, United Kingdom

E-mail address: g.elek@lancaster.ac.uk 